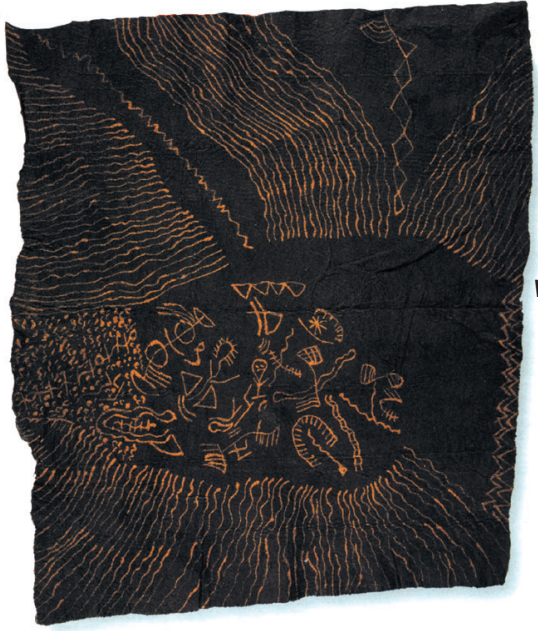

Obwohl der Umgang mit Stoffen dem Menschen selbstverständlich ist, tritt diese Beziehung selten in den Mittelpunkt öffentlicher Diskussion. Ein Grund hierfür könnte gerade in der Alltäglichkeit des Umgangs mit Stoffen liegen. Solange Stoffe die ihnen zugedachte Funktion erfüllen, wecken sie weder Kritik noch Neugierde. Allenfalls bestimmte "Schadstoffe des Monats" werden thematisiert. Ein anderer Grund liegt wohl darin, daß die Vielfalt der Stoffe und ihrer Wirkungen eine solche Unübersichtlichkeit geschaffen hat, daß eine bündelnde Perspektive der Beschreibung bisher nicht aussichtsreich erschien.

Das Konzept der Stoffgeschichten könnte die Leerstelle einer präzisen Beobachtung und Beschreibung des Umgangs mit Stoffen füllen. Denn zum einen verbindet sich mit diesem Konzept eine wissenschaftliche Programmatik, zum anderen eine gesellschaftliche Aufgabe - nämlich das Erzählen einer Geschichte, die soziales Handeln strukturiert und damit eine Vermittlungschance im Spannungsfeld zwischen Wissenschaft, Technik, Politik und Öffentlichkeit eröffnet.

\title{
Stoffgeschichten - eine neue Perspektive für transdisziplinäre Umweltforschung
}

Stefan Böschen, Armin Reller und Jens Soentgen*

\section{Ein Konzept - zwei Perspektiven}

Der Umgang mit Stoffen wandelte sich durch die Etablierung der chemischen Industrie in der zweiten Hälfte des 19. Jahrhunderts erheblich. Es wurden nicht nur ganz neue, synthetische Stoffe in wachsender Zahl hergestellt, sondern die Produktion von Stoffen in bis dahin unvorstellbaren Mengen ermöglicht. Die Kehrseite dieser Entwicklung blieb nicht lange verborgen. Streitigkeiten auf Grund der konkurrierenden Naturnutzung durch andere Wirtschaftszweige zeigten, daß im "Stoffumsatzgewerbe" nicht nur Stoffe, sondern auch Schadstoffe entstanden. Deren Regulierung wurde schon bald notwendig. Im Zusammenhang mit der Umsetzung von Schutzmaßnahmen bildete sich eine wirkungsvolle Arbeitsteilung zwischen Wissenschaft, Staat und Industrie heraus und blieb in dieser Form lange Zeit unangefochten ${ }^{[1]}$. Der aufkommenden Umweltbewegung wurde diese eingespielte Praxis des Umgangs mit Stoffen zunehmend problematisch.

*Postadresse : Dr. J. Soentgen

Wissenschaftszentrum Umwelt (WZU)

Universität Augsburg

Universitätsstraße 1

D-86159 Augsburg (Deutschland)

E-Mail: soentgen@wzu.uni-augsburg.de
Vor dem Hintergrund einer Reihe von spektakulären Unfällen und Katastrophen (Seveso, Bhophal und andere) begann schließlich in den 1970er und 1980er Jahren eine generelle Chemiediskussion, die zu einer Reflexion auf die Chemie, ihre Grundlagen und gesellschaftlichen Implikationen beitrug. Wie weitreichend diese Infragestellung war, zeigte sich nicht zuletzt in der Diskussion um grundlegende Perspektiven einer Chemiepolitik ${ }^{[2]}$. Im Zuge dieser Auseinandersetzungen wurde der Umgang mit Chemikalien ganz neu strukturiert. So wichtig diese Debatten waren, so verengte sich in ihnen jedoch tendenziell der Blick auf Gefahrstoffe und das Management ihrer Ströme. Generelle Perspektiven für den gesellschaftlichen Umgang mit Stoffen fehlen bis heute, die Einbettung von Stoffen in gesellschaftliche Handlungszusammenhänge und Praxisdomänen ist bisher kaum untersucht worden. Die Frage, inwiefern experimentell spezifizierte Stoffeigenschaften in technisch und praktisch nutzbare StoffFunktionen übersetzt werden können, geht über die Kompetenz der Chemie als Naturwissenschaft hinaus. Um hier weiterzukommen, scheint uns ein Perspektivenwechsel von dem mathematisierten Stoff(strom)management $\mathrm{zu}$ Stoffgeschichten, die über den Umgang mit Stoffen erzählen, sinnvoll.
Wie weitreichend ein solcher Perspektivenwechsel ist, zeigt ein Blick auf die Entwicklung und den Stand der Umweltforschung. Unter ökosystemarem Blickwinkel formulierte diese in den frühen 1970er Jahren ihre Programme und lief damit in ein Dilemma hinein. Denn mit der Beschreibung der Umweltwirkungen als Eingriffe in Ökosysteme wurde eine Beschreibungssprache gefunden, die den technischen Zugriff auf Natur, welcher der modernen Wissenschaft seit ihren Anfängen eigen ist ${ }^{[3]}$, verlängert. Dabei hatte doch gerade die technische Nutzung der Natur die beobachteten Probleme erzeugt. Kann eine Problemlösung, die den gleichen Denkmustern entspringt wie die Problemursache, überhaupt eine Lösung sein? Abhilfe wurde unter anderem in einer transdisziplinären Reformulierung der Programmatik gesucht, und dazu wurden auch Institutionen eingerichtet, zum Beispiel im Kontext der Klimawandelforschung. Auffällig bleibt aber auch hier die Diskrepanz zwischen programmatischen Entwürfen einerseits und der Praxis der Umweltforschung anderseits. Denn vielfach ist die Umweltforschung von Detailanalysen beherrscht und kaum anschlußfähig an politisch-öffentliche Entscheidungsprozesse $^{[4]}$. Oder sie verlängert weiterhin die technokratische Perspektive, wie es sich zum Beispiel sehr prägnant in der 
umweltwissenschaftlichen Programmatik Hans-Joachim Schellnhubers (Potsdam Institut für Klimafolgenforschung) zeigt, der nichts Geringeres als die zweite Kopernikanische Revolution ausruft. ${ }^{1)}$

Somit besteht eine doppelte Herausforderung: Zum einen bedarf es übergreifender Konzepte, die den Anspruch auf Transdisziplinarität der Umweltforschung einzulösen vermögen; zum anderen bedarf es einer Forschung, deren Resultate nicht nur innerakademisch kommuniziert werden können. Das Konzept der "Stoffgeschichte", das wir unter Weiterführung der Arbeit von Huppenbauer und Reller ${ }^{[6]}$ hier vorstellen wollen, scheint die dargelegten empirischen wie konzeptionellen Herausforderungen behandeln zu können. Denn mit diesem Konzept wird - auf der empirischen Ebene - der Blick auf den gesellschaftlichen Umgang mit Stoffen von den Verengungen der Management-Perspektive befreit und neu justiert. Des weiteren werden - auf der konzeptionellen Ebene - die Herausforderungen an eine transdisziplinäre Umweltforschung aufgegriffen und produktiv gewendet. Stoffgeschichten sind nicht nur eine Heuristik zur differenzierten Beschreibung des gesellschaftlichen Umgangs mit Stoffen, sondern auch eine Stoffgeschichte, eine Erzählung virtueller oder faktischer Natur. Als solche kann sie als Vermittlerin zwischen den unterschiedlichen Teilsystemen der Gesellschaft wirken. Vor diesem Hintergrund muß die doppelte Perspektive des "Stoff-Blicks" und der "Stoff-Erzählung" als ein zentrales Merkmal angesehen werden. Diese beiden Perspektiven sollen im folgenden verdeutlicht und entwickelt werden.

\section{Kontextualisierung: Perspektive des "Stoff-Blicks"}

Bei der Analyse von gesellschaftlich thematisierten Problemen kann die Wissenschaft nicht mehr selbstverständlich davon ausgehen, unbestrittener Sachwalter des Wissens zur Beschreibung und Bearbeitung solcher Probleme zu sein. Andere Wissensakteure aus verschiedenen institutionellen Bereichen der Gesellschaft werden für die Definition des relevanten Wissens zunehmend wichtig. Die Kontexte beginnen zu sprechen und bedürfen einer systematischen Berücksichtigung ${ }^{[7]}$. Unserer Auffassung nach bietet sich als erster, aussichtsreicher Schritt die Öffnung von Forschung für eine Erzählperspektive an. Eine solche Entwicklung ist alles andere als selbstverständlich, kann doch die Entwicklung neuzeitlicher Wissenschaft als Prozeß der "Reinigung" von allen narrativen Elementen beschrieben werden. ${ }^{2}$ Nun ist aber gerade im Zusammenhang mit dem Umweltdiskurs die konstitutive Bedeutung von "Narrationen" für die Entwicklung gesellschaftlicher Problembereiche aufgezeigt worden (für das Beispiel "Klima" ${ }^{[9]}$ ). Solche Narrationen strukturieren die öffentliche Auseinandersetzung und setzen zugleich den Rahmen für spezifische wissenschaftliche Perspektiven zur Beschreibung der Problemlage.

\subsection{Stoffgeschichten als Heuristik}

Um die Bedeutung von Stoffen zu untersuchen, ist nicht allein ihre chemische Beschreibung nötig, sondern ebenso die Analyse der unterschiedlichen Praxisdomänen und Diskurse, in denen Stoffen eine je kontext- beziehungsweise diskursspezifische Bedeutung zuerkannt wird. Daraus ergibt sich eine Abgrenzung: Das Konzept der Stoffgeschichten schließt nicht an dasjenige vom Stoffwechsel an, das ausgehend von der Marxschen Analyse in der Hauptsache an der Formung von Natur durch menschliche Arbeit ansetzt - und damit spezifische Verkürzungen des komplizierten Wechselspiels zwischen Stoffen und Gesellschaft in Kauf nimmt. Denn hinter der heute so geläufigen Metapher vom Stoffkreislauf steht eine Vorstellung, welche die Natur als eine Art vergrößerte Fabrik sieht, in der die Stoffe von einem Reservoir zum anderen wandern ${ }^{[10]}$. Zudem wird dabei als dominante Art des Umgangs mit Stoffen die Arbeit angesehen. Doch die Vielfalt des Wechselspiels zwischen Stoffen und Gesellschaft geht über den Inhalt des klassischen, an der Produktion von Gütern und Waren orientierten Arbeitsbegriffs hinaus. Auch kommunikative Prozesse beeinflussen den gesellschaftlichen Umgang mit der Natur. Ein praxistheoretischer Ansatz ist hier weiterführend, in welchem »die einzelne Handlung als Teil von sozialen Handlungsgefügen, von gemeinsamen, sozialen Praktiken ${ }^{[11]}$ verstanden wird.

Dabei war und ist die neuzeitliche Wissenschaftspraxis dadurch gekennzeichnet, die Praxisdomänen begrenzt zu halten. Diese scharfe Grenzziehung ist einerseits funktional, anderseits führt sie zu einem "Laborblick" auf die Phänomene. Die Naturwissenschaft beobachtet also nicht "die Natur", sondern eine zweite, von ihr selbst erzeugte Natur, die sich den Anforderungen des Laboratoriums fügt ${ }^{[12]}$. Bruno Latour ${ }^{[13]}$ prägte hierfür den Begriff der "kahlen Objekte", welche sich durch die weitreichende Kontrolle der Randbedingungen ihrer Erzeugung auszeichnen. Die wissenschaftssoziologische Diskussion hat freilich deutlich gemacht, daß in der Wissenschaft selbst anderen Wissensformen (vor allem implizitem Wissen oder Erfahrungswissen) eine erhebliche Bedeutung zukommt. ${ }^{3)}$

Ebenso läßt sich auf der Ebene wissenschaftlicher Konzeptionen zeigen, daß diese nicht allein einem wissenschaftlichen Diskurszusammenhang entspringen, sondern vielfach in Wechselwirkung mit übergeordneten Diskursen innerhalb der Gesellschaft entstehen. Ludvik Fleck ${ }^{[17]}$ geht sogar so weit, diesen Prozeß als konstitutiv für Wissenschaft anzusehen. Denn Einfachheit wie auch Klarheit des wissenschaftlichen Wissens entsteht durch öffentliche Vermittlung von Forschungsergebnissen; "populäres Wissen" ist deshalb eine wesentliche Form wissenschaftlichen Wissens. Anders gesagt: Wissenschaftler brauchen Geschichten, um sich ihrer Erkenntnisse zu vergewissern. Zugleich ist die Erzählform die Struktur, in welcher Wissen in öffentlichen Diskursen auftaucht. Somit kommt Erzählungen eine zweifache Aufgabe zu: sie erzeugen zum einen Anschlußfähigkeit $\mathrm{zu}$ den verschiedenen außerakademischen Bereichen; zum anderen

1) Dabei läßt er nicht unbedingt demokratietheoretische Sensibilität erkennen, wenn er schreibt: »Global telecommunication will ultimately establish a cooperative system generating values, preferences and decisions as crucial commonalities of humanity online. « ${ }^{[5]}$ Damit wird zum einen eine Vereinheitlichung der Werte unterstellt, wie sie gerade für plurale Demokratien untypisch ist, und zum anderen werden die typischen und notwendigen Auseinandersetzungen und Konflikte zwischen den Kulturen unterschlagen.

2) Entsprechend erlangte die methodisch angeleitete und experimentell verfahrende Naturforschung gegenüber der Naturgeschichte einen immer größeren Stellenwert und löste diese zu Beginn des 19. Jahrhunderts ab [8].

3) Diese schon immer stattfindende "verdeckte Kontextualisierung" zeigt sich unter anderem an der Bedeutung von "implizitem Wissen" oder "Erfahrungswissen". Mit dem Topos des tacit knowledge [14] wird die Bedeutung von Knowhow, technischen Tricks sowie eines Gefühls für Regeln und deren Anwendung hervorgehoben. Klassisch ist die Studie von Harry Collins [15] über Versuche zum Nachbau des TEA-Lasers. Sie zeigte, daß allein denen der Nachbau des Gerätes gelang, die in direktem Kontakt mit den Erfindern standen. Verschriftlichtes Wissen war nicht ausreichend. Entsprechend definiert Collins tacit knowledge als: »knowledge or abilities that can be passed between scientists by personal contact but cannot be, or have not been, set out or passed on in formulae, diagrams, or verbal descriptions and instructions for action.«[16]. 
unterstützen sie eine "Selbstdisziplinierung zum Kontext". Sie leiten Wissenschaftler dazu an, die möglichen Anwendungsbezüge des erzeugten Wissens mit zu reflektieren.

\subsection{Konkretisierung der kontextualistischen Wissenschaftsperspektive}

Bereits bei der Konstruktion von Tatsachen spielen Erzählungen eine beträchtliche Rolle. Diese wird um so bedeutender, je stärker das Themenfeld mit gesellschaftlichen Problemdiskursen interferiert. Denn hier werden nicht nur die relevanten Problemperspektiven verhandelt, sondern ebenso die möglichen Lösungsmuster - und die unterscheiden sich je nach Perspektive erheblich. Eine gute "Problemgeschichte" muß deshalb eine Diagnose der Situation hergeben, die Dringlichkeit des Problems unter-

4) Ein solcher Prozeß der Kontextualisierung läßt sich zum Teil schon an Einsichten der Ökologischen Chemie studieren. Sie machte deutlich, daß es unmöglich sei, die Vielfalt möglicher Wirkungen von Chemikalien vollständig aufzuschlüsseln. Jüngst wurde deshalb vorgeschlagen, die schadensbezogene Sichtweise zu überschreiten und auf Gefährdungen umzustellen. Chemikalien sollten als regulationsbedürftig gelten, wenn sie ein gewisses Gefährdungspotential aufweisen.

Dieses wiederum kann durch allgemeine Kriterien beschrieben werden, wie zum Beispiel Persistenz und Reichweite [22]. Mit diesen Kriterien hat das Vorsorgeprinzip nun Eingang in die Neuordnung der europäischen Chemikalienpolitik gefunden.

5) Sherwood Rowland, einer der Erfinder der FCKW-Ozonzerstörungshypothese, bemerkte viele Jahre später: »In retrospect, the main advance was to get out of the lab and into the real world by following a molecule from its release into the atmosphere to its eventual destruction many years later.« [23]

6) Ein Fallbeispiel, das in diesem Zusammenhang instruktiv sein könnte, ist die Anwendung von Platin-Katalysatoren bei Fahrzeugen, die mit bleifreiem Benzin fahren. Hierzu wurden diverse Risikohypothesen formuliert. Eine davon besagte, daß es zu einer verstärkten Bildung von Salpetersäure in der Umwelt kommen würde. Grundlage für dieses Argument war die Verwendung von Platin als Katalysator bei Herstellungsverfahren dieser Säure. Allerdings sind dabei die Randbedingungen deutlich andere (viel höherer Druck und

Temperatur), so daß diese Hypothese zurückgewiesen werden konnte. Jedoch gewinnt in der jüngsten Zeit eine andere Risikohypothese an Bedeutung. Es wurde entdeckt, daß Nano-Pt wasserlöslich ist. Dies widerspricht allen bisherigen Vorstellungen über Stabilität und Löslichkeit von Edelmetallen. Somit kann Platin in Form kleinster Partikel bioverfügbar werden und möglicherweise in Organismen als Biokatalysator wirken. Die Effekte sind noch unerforscht, könnten aber ein hohes Risikopotential bergen. Auch in diesem Fall hat der Anwendungskontext ganz besondere Stabilitätsbedingungen, die durch Analogien aus dem Laborkontext nicht erfaßt wurden. streichen und schließlich bestimmte Therapeutika anbieten können, mit denen Gesellschaften sich des diagnostizierten Problems erwehren können. Bisher wurden die relevanten "Problemgeschichten" durch die Naturwissenschaft definiert und entsprechende technische Lösungsmuster angeboten. Jedoch hat sich inzwischen diese Situation verändert. Durch die Umwelt- und Risikodiskurse öffnete sich das Feld wissenschaftlicher Wissensproduktion zur (politischen) Öffentlichkeit ${ }^{[18]}$. Von den verschiedensten Standorten innerhalb der Gesellschaft wird gesprochen, eine "Begrenzung der sprechenden Subjekte" [19] ist kaum mehr möglich. Aus den ehedem "kahlen Objekten" entstehen "Risiko-Objekte" [13] oder "extended facts" [20], bei denen Akteure mit ganz unterschiedlichen Perspektiven an der Konstruktion der Objekte mitwirken.

Das Konzept der Re-Kontextualisierung eröffnet hier neue Wege. Diese Idee wurde am Beispiel der Gentechnologie schon von Wolfgang Bonss, Rainer Hohlfeld und Regine Kollek in die Diskussion eingebracht ${ }^{[21]}$. Re-Kontextualisierung stellt sich dabei als eine Strategie der "reflektierten Transzendierung des Laborkontextes" - also eine Anreicherung "kahler Objekte" - und letztlich als "Vergesellschaftungsform wissenschaftlichen Wissens" [21a] dar. Wichtige Anregungen und Perspektiven erhält eine Heuristik der Kontextualisierung mithin aus der Rekonstruktion von pragmatischen und semantischen Kontexten der komplexen Ausgangsphänomene. In der letzten Konsequenz führt ein so verstandener Kontextualismus zu einem wissenschaftspolitischen Programm, weil »die Schritte der Erweiterung des Laborkontextes und die Bedingungen der Anwendung selbst zum Forschungsgegenstand gemacht werden. «[21a] Ausgangspunkt ist zwar wissenschaftliches Wissen, jedoch wird es in seinen Wechselwirkungen mit der Gesellschaft aufgeschlüsselt. ${ }^{4)}$

Hier ist ein Blick in die Welt der Chemie hilfreich. Sie liefert eine Fülle von Beispielen dafür, wie die Perspektive systematisch auf "kahle Objekte" begrenzt wurde. Ebenso kann man erfahren, welche besonderen Herausforderungen sich bei der Konstruktion von "RisikoObjekten" stellen. Bisher beruhten die Sicherheitsvorstellungen in der Chemie auf Konzepten von "Stabilität" und "Inertheit" oder aber kontrollierbarer Reaktivität, die sich aus gewissen chemischen Parametern der jeweiligen Stoffe bestimmen ließen. Ziel der Chemie war ja die Herstellung von Stoffen, die eine möglichst ausgeprägte Stabilität oder Wirkungsweise für spezifische Funktionen auszeichnet. Diese Zuschreibung hat sich in einigen Fällen als Fehlschluß erwiesen, wobei das Fallbeispiel der Fluorchlorkohlenwasserstoffe FCKW sicherlich $\mathrm{zu}$ den prominenteren gehören dürfte. Diese rein synthetische Stoffklasse wurde wegen klar spezifizierter chemischer ("Stabilität") und physikalischer (durch die Zusammensetzung einstellbarer Siedepunkt) Eigenschaften als das ideale Kühlmedium entwickelt und in großem Maße eingesetzt. Unter den Bedingungen der Troposphäre sind diese Stoffe auch stabil (man schloß daraus, daß sie inert seien), können jedoch von den UVStrahlen in der Stratosphäre aufgebrochen werden (sie sind also nicht stabil unter den Strahlungsbedingungen der Stratosphäre). Mit der Stratosphäre kam ein anderer pragmatischer Kontext in den Blick. Dies war die Voraussetzung, die Fiktion der Inertheit in diesem Fall zu "enttarnen".5) Solange die Stabilität allein von der Wissenschaft definiert wurde, war es eher wahrscheinlich, "diskursive Schläfer" zu erzeugen, also solche Stoffe, bei denen man von einer Sicherheit ausging, die sich im nachhinein als unbegründet herausstellte. Denn im Rahmen herkömmlicher wissenschaftlicher Praxis wurde relativ umstandslos aus der Stabilität von Stoffen im Laborkontext auf ihre Inertheit geschlossen, ohne jedoch die unterschiedlichen Anwendungskontexte und Wirkungsfelder zu kennen. ${ }^{6}$ Das Leitbild "klassischer Naturforschung" [24] sah dies freilich erst gar nicht vor.

Zur näheren Analyse und Differenzierung von "Stoffgeschichten", die sich als komplementäre Ergänzungen zur wissenschaftlichen Beschreibung der Stoffe auffassen lassen, würde es sich anbieten, "Leitstoffe" zu identifizieren: Sie sollten entweder eine Konkurrenz unterschiedlicher Stoffpraxen abbilden oder - und damit kontrastierend - das Monopol einer wissenschaftlichen Praxis widerspiegeln. Unter die erste Gruppe fallen beispielsweise die Farbstoffe, bei denen es vor deren wissenschaftlicher Entschlüsselung vielerlei Rezepte und Prozeduren der Herstellung gab. Zu der zweiten Gruppe zählen beispielsweise die Silicone, eine vollkommen synthetische Substanzklasse, von der man annahm, daß sie unter gewöhnlichen Bedingungen unzugänglich für Reaktionen sei ${ }^{[25]}{ }^{7)}$ Die "Inert-Fiktion" war und ist ihrerseits so stabil, daß man diese Substanzklasse in der Zwischenzeit in über 14000 Alltagsprodukten auf den Markt gebracht hat. Jedoch läßt sich die Ver- 


\section{Exkurs}

\section{Stoffe und ihre Wirkungsformen in Raum und Zeit: Das Beispiel der Silicone}

Die Nutzung von Stoffen geht in der Regel mit der Umgestaltung ihrer Zusammensetzung und ihrer Erscheinungsform einher. Je nach den Erfordernissen werden mehr oder weniger komplizierte Abfolgen von chemischen und physikalischen Prozessen zur Umwandlung, Umformung und Umverteilung von Stoffen verwendet. Ziel ist die Herstellung von Stoffen mit spezifischen Wirkungsformen, die dann eine Funktion erfüllen können. So werden aus - hier im Bild unten links gezeigtem - Quarz oder Bergkristall (Siliciumdioxid, $\mathrm{SiO}_{2}$ ) mit hohem Energieaufwand das für die Chip-Industrie unersetzbare Halbleitermaterial Silicium, aber auch die im Alltag weitverbreiteten Silicone und Siloxane hergestellt. Aus einem Rohstoff entstehen so Stoffe mit sehr unterschiedlichen Wirkungsformen. Solche Stoffe kann man auch als portionierbare Technologien bezeichnen, da sie für die Erfüllung bestimmter Funktionen gestaltet wurden. Wie jedes andere funktionelle Material auch, haben sie bestimmte Nebenwirkungen: Da Silicone und Siloxane in sehr vielen Haushalts- und Pflegemitteln eingesetzt werden, gelangen sie vorerst unbemerkt in Müllverbrennungsanlagen und Klärwerke. Diese beiden zweckmäßigen Einrichtungen verwerten die teils zu Biogas umgewandelten Abfalloder Reststoffe in Gasmotoren. Neben den wie geplant erwarteten Kohlenstoffverbindungen finden auch die genannten Siliciumverbindungen ihren unvorhergesehenen Weg in den Verbrennungsraum des Motors. Im Gegensatz zu den Kohlenstoffverbindungen ("Biogas"), die zu gasförmigem Kohlendioxid und Wasser verbrennen, werden sie zu - im Bild unten rechts gezeigten - festen Ablagerungen von Siliciumdioxid $\left(\mathrm{SiO}_{2}\right.$, Quarz) umgewandelt, die sich bereits im Motorraum oder auf nachgeschalteten Filtern und Katalysatoren absetzen und die Funktion des Gasmotors massiv beeinträchtigen oder blockieren. Solche Überraschungsmomente kommen in Stoffgeschichten häufiger vor: Der Protagonist taucht in Zusammenhängen auf, in denen man ihn nicht vermutet hat.

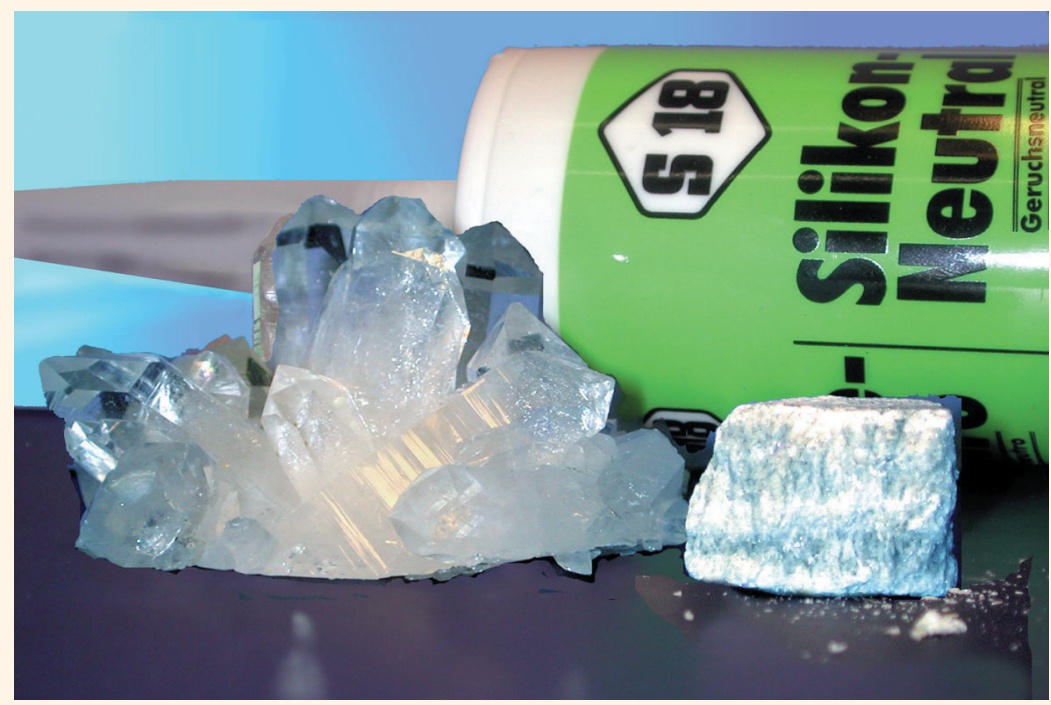

mutung nicht ohne weiteres von der Hand weisen, daß auch in diesem Fall die unter Laborbedingungen realisierte Stabilität der Substanzklasse nicht als Inertheit interpretiert werden dürfte. Denn manche Mikroorganismen lernen, diese Stoffe in ihren Stoffwechsel zu integrieren. Die Silicone könnten daher unter Umständen zu einem weiteren Beispiel in der Nachfolge von DDT und FCKW werden, bei denen zunächst unerwünschte Wechselwirkungen im $\mathrm{Zu}$ sammenhang mit anderen Kontexten von vornherein ausgeschlossen worden waren.

\section{Stoffgeschichten als "Stoff-Erzählung"}

Der einflußreiche russische Avantgardist Sergej Tretjakow regte schon in den zwanziger Jahren des letzten Jahrhunderts "Biographien von Dingen" an ${ }^{[26]}$. Er nahm daran Anstoß, daß der "bürgerliche Roman" einzelne Helden allzusehr in den Mittelpunkt stelle. In einer Umkehrung empfahl der Marxist, es doch einmal anders $\mathrm{zu}$ versuchen, und nicht Menschen, sondern objektive Dinge wie etwa den Wald, das Brot, die Kohle, das Eisen, den Flachs, die Baumwolle oder das Papier zu beschreiben, welche durch die gesellschaftlichen Formationen wandern und allmählich zu "nützlichen Dingen" werden. Anfang der dreißiger Jahre erschienen einige Bücher, die das von Tretjakow entworfene Programm verwirklichten, und 1934 das erste, das seine Struktur allein einer Sache verdankte: Sage und Siegeszug des Kaffees Die Biographie eines weltwirtschaftlichen Stoffes von Heinrich Eduard Jacob [27]. Im Prolog schreibt der Autor: »Nicht die Vita Napoleons oder Cäsars wird hier erzählt, sondern die Biographie eines Stoffes. Eines tausendjährigen, treuen und machtvollen Begleiters der ganzen Menschheit. Eines Helden.« Und gegen Ende seiner eindrucksvollen Geschichte erzählt Jacob auch noch, wie er, in Rio de Janeiro unterwegs, einen Kaffeezweig betrachtete und einen Tagtraum hatte: Er gehe an einem breiten, gelben Fluß stromaufwärts und gelange über Paris, Venedig, Wien nach "Arabien". Erwachend nimmt er sich vor, eine "Mythologie der Rohstoffe" zu schreiben.

Immerhin bezeichnet die Deutsche Biographische Enzyklopädie Jacob als Begründer des modernen Sachbuchs. Sein Werk über den Kaffee liest sich wie ein Roman, bietet dem Leser indes auch Reportagephotos und ein ausführliches Literaturverzeichnis, was die Mischform unterstreicht. Aktuellere Beispiele für "Stoffbiographien" sind etwa Jürgen Dahls Buch Aufschlüsse: Kalkstein, Feuerstein, Schiefer - drei Versuche zur Geologie ${ }^{[28]}$ oder das von Philip Ball $\mathrm{H}_{2} \mathrm{O}$ : Biographie des Wassers ${ }^{[29]}$. Für das Projekt "Stoffgeschichten" läßt sich aus diesen Büchern vieles lernen. Sie nehmen die Aufgabe ernst, zu erzählen: sie bringen stoffbezogene Themen in eine zirkulationsfähige Form. Ihnen gelingt, was auch das hier vorgestellte Konzept beabsichtigt: Aufklärung über Stoffe zu vermitteln. Im Fokus dieser Stoffgeschichten steht allerdings fast immer "der Stoff selbst", wie er sich aus der Perspektive bestimmter Naturwissenschaften darstellt. Die gesellschaftliche Bedeutung und Anwendungszusammen-

7) Silicone sind sogenannte siliciumorganische Verbindungen. Als Besonderheit weisen sie eine Silicium-Kohlenstoff-Bindung auf, die so in der Natur keine Analoga hat. Durch dieses Strukturmerkmal ist der Aufbau dieser Stoffe gewissermaßen teils anorganischer, teils organischer Natur, wobei diese Kombination für die ganz besonderen Eigenschaften maßgeblich ist. So sind Silicone viel wärmebeständiger im Vergleich zu herkömmlichen Kohlenstoffpolymeren und können zugleich flexibler verarbeitet werden, weil sie sich einerseits als Flüssigkeiten, anderseits als verformbare, plastisch-elastische Massen verhalten [25a] 
hänge werden nicht systematisch mitreflektiert. Damit geht ein Teil der Wirklichkeit eines Stoffes verloren. Seine Form und seine davon abhängigen Wirkungsweisen in unterschiedlichen zeitlichen und räumlichen Kontexten werden eher zufällig beachtet, nur die ihm zugedachte Funktion ist von Bedeutung. Hingegen fordert der Kontextualismus ja gerade eine Ausweitung des Blickes hin auf die semantischen und pragmatischen Kontexte des gesellschaftlichen Umgangs mit Stoffen. Es bedarf also der Verbindung der naturwissenschaftlich erarbeiteten Fakten mit geistes- und sozialwissenschaftlichen Kenntnissen und Methoden. Erst aus der fruchtbaren Verbindung der unterschiedlichen Perspektiven erwächst der "Stoff" für eine Stoffgeschichte.

\subsection{Was erzählt eine Stoffgeschichte?}

Eine Stoffgeschichte verfolgt einen Stoff nicht nur durch bestimmte chemische Konstellationen und Prozesse hindurch, sondern durch verschiedene Zeiten und Situationen. Sie zeigt, um das Beispiel der Kaffeegeschichte des Eduard Jacob nochmals in Erinnerung zu rufen, die Kontexte der Entstehung des Stoffes, und verfolgt ihn durch kulturelle und politische Szenenwechsel hindurch bis in die Gegenwart. Von einer life-cycleAnalyse, die ebenfalls Stoffe (oder Produkte) durch verschiedene Kontexte hindurch verfolgt, unterscheidet sich eine Stoffgeschichte in drei Aspekten. Zum einen dadurch, daß sie nicht nur ökonomische Kontexte berücksichtigt, sondern auch kulturelle Aspekte des Umgangs mit einem Stoff ${ }^{[30]}$. So berichtet Jacob in seinem Kaffeebuch auch über diverse Trinkkulturen und befaßt sich eingehend mit den Berliner Kaffeehäusern. Weiter hat eine Stoffgeschichte nicht das Ziel, konkrete Indikatoren $\mathrm{zu}$ errechnen, die eine Bewertung eines bestimmten Produkts oder Produktionsweges erlauben. Vielmehr geht es darum, Verständnis für Zusammenhänge zu wecken. Schließlich richtet eine Stoffgeschichte das Augenmerk nicht auf kurzlebige ökonomische Zyklen, sondern öffnet den Blick von vornherein für größere historische Zeiträume - wie ja auch Jacob sein Buch mit einer Phantasie über die erste Entdeckung der Wirkung der Kaffeebohne beginnt.

Bei einem so weit gefaßten Themenfeld stellt sich unweigerlich das Problem der Auswahl. Eine Stoffgeschichte kann natürlich nicht den Anspruch haben, "alles" über einen Stoff zu er- zählen. Andererseits darf sie auch nicht nur eine Plauderei sein, die einzelne Anekdoten aneinanderreiht, sonst landet man bei den "kleinen Kulturgeschichten", die weniger aufklären wollen, sondern eher unterhalten ${ }^{[31]}$. Es gibt hier keine Patentrezepte, wohl aber Beispiele, an denen man sich orientieren kann. Den genannten Büchern von Jacob oder von Dahl gelingt es, eine zusammenhängende Geschichte zu erzählen, die dem Leser ein Bild von dem Stoff, nicht nur eine Summe von Einzelheiten vermittelt und insofern vollständig ist. Mit anderen Worten: Über die Vollständigkeit einer Stoffgeschichte entscheidet nicht eine außerhalb ihrer liegende "objektive Sache", sondern der Leser, dessen Fragen beantwortet werden oder nicht.

Stoffgeschichten sind also keineswegs zeitlos, sondern auf ein bestimmtes Publikum bezogen. Sie sind eine Erzählung im Sinne der alten Rhetoren: Die narratio, die sich immer auch an der opinio, der Voreinstellung des Publikums, orientiert, soll unterhalten, belehren und zur Handlung motivieren - unbedingt zu vermeiden ist das taedium, die Langeweile des Publikums. (Brevitas, Kürze ist übrigens nach Quintilian eine Grundtugend der narratio, sie muß keineswegs "vollständig" sein ${ }^{[32]}$.) Gemessen an den mit Zahlen, Flußdiagrammen und Szenarios bewehrten Formen, in denen die Resultate von Umweltforschung üblicherweise dargestellt werden, mögen Stoffgeschichten zunächst einmal recht unscheinbar daherkommen. Vielleicht erscheinen sie harmlos, rein belletristisch oder nicht ganz ernst zu nehmen. Stoffgeschichten sind, vom Standpunkt der etablierten Umweltforschung gesehen, durchaus angreifbar. Sie wollen diese Umweltforschung auch nicht ersetzen, sondern ergänzen. Denn die üblichen, wissenschaftlich anerkannteren Formen, umweltrelevantes Wissen darzustellen, verlieren sich oft in Details - und man

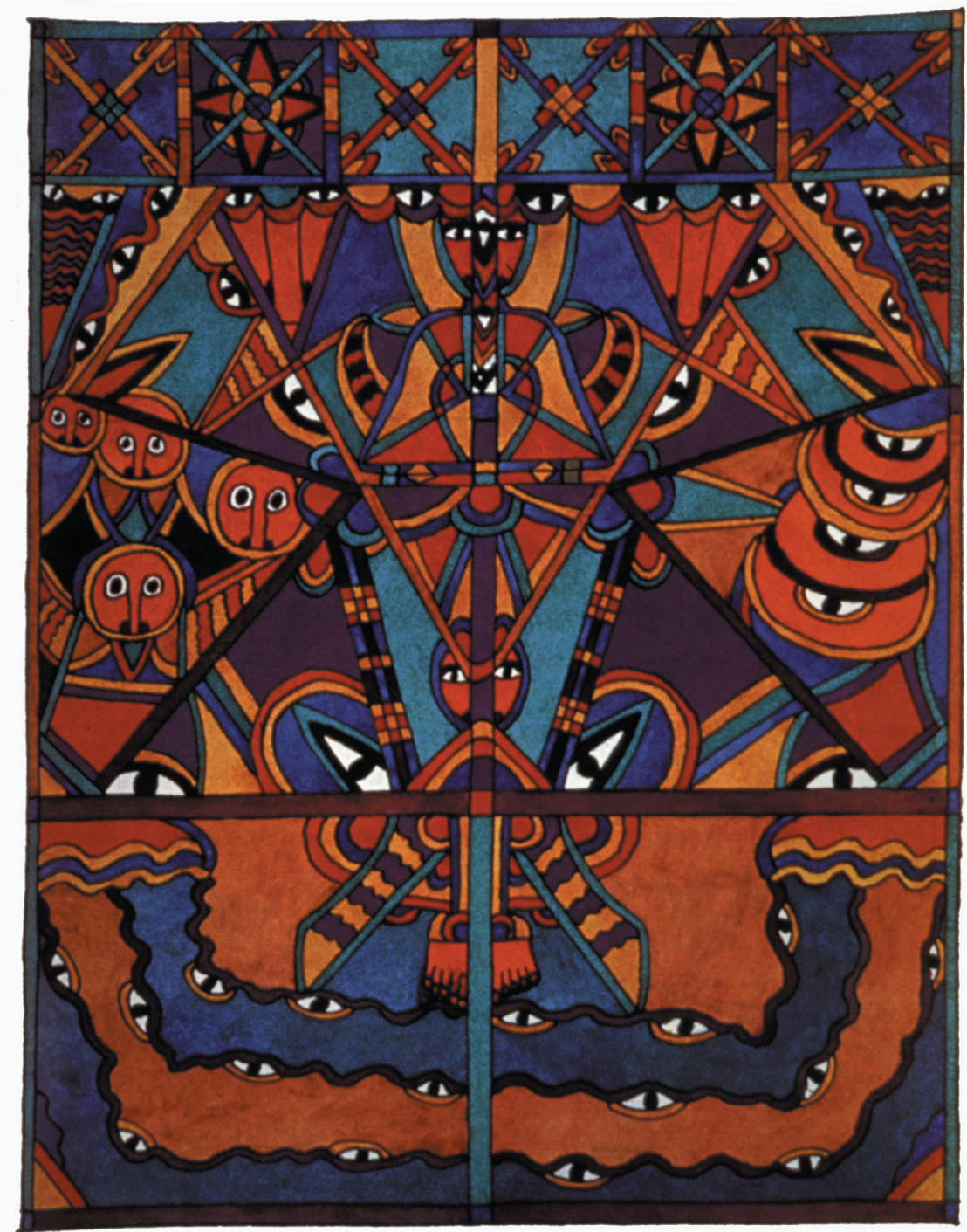

Gewebte Stoffe können viel erzählen. 
sieht bald den Wald vor lauter Bäumen nicht mehr. Eine Stoffgeschichte hingegen vermittelt einen Blick auf das Ganze, und zwar ohne jene plakativen Metaphern, die in der populären ökologischen Diskussion so beliebt sind ("ökologischer Fußabdruck", "Stoffkreislauf", "ökologischer Rucksack"). Zugleich gehen sie von ausschnitthaften, mehr oder weniger willkürlich gewählten Zeitfenstern weg und wagen eine weiter ausgreifende Sicht. So können Entwicklungen sichtbar werden, die bei den präziseren, kürzer greifenden Analysen, die etwa im Zusammenhang des Stoffstrommanagements üblich sind, nicht erfaßt werden. Insgesamt kann, so meinen wir, durch Stoffgeschichten das Verständnis für stoffliche Prozesse vertieft werden.

Auch wird die Phantasie angeregt: Denn der Blick über die kleinen Einheiten der supply-chains und der sogenannten life-cycles (die das "Leben" eines Stoffes keineswegs ausschöpfen) gibt ein Gespür für die Potentiale, die in einem Stoff stecken. Geschichten regen die Phantasie an. Die Gegenwart von Stoffen an erwünschten oder unerwünschten Orten läßt an neue Landkarten denken, Stofflandkarten, auf denen sich ihre Herkunft und Verbreitung, Wirkungsformen und Effekte in Raum und Zeit verfolgen lassen ${ }^{[33]}$.
Darüber hinaus haben Stoffgeschichten eine wichtige kommunikative Funktion: Sie können stoffbezogenes Wissen in eine literarische Form bringen, welche es vermag, die Wände des Potentialtopfs, innerhalb dessen üblicherweise die Kommunikation über Stoffe und Materialien kreist, zu durchtunneln. So können Geschichten über Stoffe, die in der Öffentlichkeit bisher nur ein exotisches Nischenthema sind, was ihrer tatsächlichen Bedeutung für die Reproduktion der Gesellschaft keineswegs entspricht, sich in die öffentliche Diskussion einhaken: Eventuell kann sich sogar ein regelrechter Diskurs formieren.

Die Auswahl der Stoffe wird nicht durch ein wissenschaftlich vorgeprägtes Suchmuster bestimmt, sondern folgt einer publizistischen Kategorie: der Aktualität ${ }^{[34]}$. Diejenigen Stoffe sollen beschrieben werden, an deren Geschichte die Gesellschaft ein begründetes Interesse hat oder doch haben sollte. Beispiele sind das Wasser, das Erdöl, "Plastik", Baumwolle, Papier, Kalkstein, Kohlendioxid, Sand, Staub und so weiter. Die Auswahl ist natürlich nicht annähernd vollständig, sie soll vor allem zeigen, daß sich der Titel einer Stoffgeschichte nicht an naturwissenschaftlich geprägten Kategorien orientiert, sondern an dem Verständnis des breiteren Publikums, für das solche Geschichten erzählt werden.

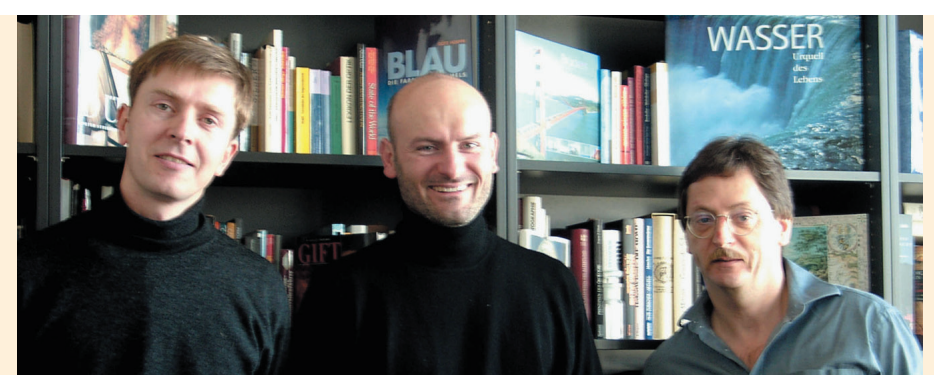

Jens Soentgen (links): Geboren 1967 in Bensberg, Nordrhein-Westfalen, studierte zunächst Chemie, promovierte aber in Philosophie, mit einer Arbeit über den Stoffbegriff (Das Unscheinbare, Berlin 1997). Lehraufträge führten ihn anschließend an mehrere Universitäten in Deutschland. Mehrfach war er in Brasilien als Gastprofessor für Philosophie tätig. Seit 2002 ist er wissenschaftlicher Leiter des Wissenschaftszentrums Umwelt (WZU) der Universität Augsburg. Zuletzt erschien von ihm das Buch Selbstdenken! 20 Praktiken der Philosophie (Wuppertal 2003).

Stefan Böschen (Mitte): Geboren 1965 in Waldshut, Baden-Württemberg. Studium des Chemie-Ingenieurwesens mit Diplomabschluß; 1995- 1998 Vertiefung in Philosophie sowie Soziologie; Promotion mir einer Arbeit zur Risikogenese, der Erarbeitung von Risiko-Wissen im Laufe der Geschichte, analysiert an vier Fallstudien. Seit 1999 an der Universität Augsburg im Sonderforschungsbereich "Reflexive Modernisierung". Er ist WZU-Mitglied und Projektleiter.

Armin Reller (rechts): Geboren 1952 in Winterthur, Kanton Zürich. 1992-1998 ordentlicher Professor am Institut für Anorganische und Angewandte Chemie der Universität Hamburg; daneben seit 1995 Leiter des Programms Solarchemie/Wasserstoff/Regenerative Energieträger (BEW, Bern). 1999 Übernahme des neueingerichteten Lehrstuhls für Festkörperchemie im Institut für Physik der Universität Augsburg. Er ist Vorstandssprecher des WZU.

Das Wissenschaftszentrum Umwelt wurde im Jahr 2000 im Rahmen der bayerischen High-Tech-Offensive gegründet. Unter dem Leitthema "Zukunftsfähiger Umgang mit Stoffen, Materialien und Energie" bündelt das WZU die Umweltforschung an der Universität Augsburg. Böschen, Reller und Soentgen arbeiten in verschiedenen Projekten im WZU zusammen.

\subsection{Wie werden Stoffgeschichten erzählt?}

Die Herausforderung für den Geschichtenerzähler liegt einmal darin, sein Publikum überhaupt für ein so sprödes Thema, wie es Stoffe in ihren erkannten, versteckten oder erdachten Wirkungsformen nun einmal sind, zu interessieren. Das ist die klassische rhetorische Herausforderung des sogenannten genus humile, jener Redegattung, die es mit gemeinhin als langweilig oder gar abstoßend wahrgenommenen Dingen zu tun hat (rhetorische Methoden, auch für solche Themen Aufmerksamkeit zu erzielen, nennt Wessel $\left.{ }^{[35]}\right)$. Die zweite Herausforderung liegt darin, die vielfältigen Fakten, welche zu einem Stoff in unterschiedlichen disziplinären Diskursen gesichert wurden, zu einer Geschichte $\mathrm{zu}$ verbinden. Es kommt hier auf die alte Kunst der synopsis, der Zusammenschau an. Wichtig ist, das geistige Band, sozusagen den Mittelbegriff zu finden, der die Einzelheiten zu einem kommunizierbaren Ganzen verbindet. Eine Technik, diese Einheit zu erreichen, welche sich in Stoffgeschichten häufig findet, ist die Personalisierung. Ein Stoff wird dabei wie eine Person dargestellt und charakterisiert. Explizit geschieht dies etwa bei Jacob, der den Kaffee im Pro$\log$ zu seinem Buch als einen "Helden" bezeichnet. Bisweilen wird die Personalisierung auch implizit vorgenommen. Sie ist übrigens keineswegs nur ein literarisches Procedere, sondern hat ein fundamentum in re: Schon eine winzige Stoffportion erzählt von den Umständen ihrer Entstehung. An ihrer Form läßt sich ihre Geschichte ablesen ${ }^{[6]}$. Stoffarten als Ganze sind darüber hinaus und durch diese Mikrogeschichten hindurch eingebunden in die Prozesse der symbolischen und materiellen Reproduktion der Gesellschaft. Ihnen wächst damit eine zweite Geschichtlichkeit zu. Es gibt daher tatsächlich eine gewisse Analogie zwischen Stoffen und menschlichen Individuen.

Auf der Personalisierung aufbauend, gibt es weitere Techniken, die in Stoffgeschichten vielfach auftauchen: Analog wie die Biographienforschung in menschlichen Lebensgeschichten "Daseinsthemen" ausmacht ${ }^{[36]}$, an denen sich eine Person abarbeitet, oder wie auch Historiker die Geschichte eines Landes oder eines Volkes durch gewisse Leitthemen strukturieren, lassen sich auch für Stoffe Leitmotive formulieren, die in der Geschichte eines Stoffes immer wieder, vielleicht in gewandelter Form, auftauchen. Beim Wasser kann man als 
ein solches Leitmotiv etwa seine natürliche Tendenz feststellen, Grenzen zu überschreiten oder diffusiv zu unterwandern ${ }^{[37]}$; für die Geschichte der Baumwolle wird der Nord-Süd-Gegensatz in seinen verschiedenen Ausprägungen ein solches Leitmotiv sein; beim Kalk sieht Dahl ${ }^{[28]}$ als Leitmotiv den Gegensatz zwischen einer Auflösungstendenz und der Fähigkeit, vorgegebene Formen mimetisch genau abzubilden.

Insgesamt läßt sich jedoch keine Anleitung für das Abfassen von Stoffgeschichten geben. Zum einen müßte zu diesem Zweck ein größerer Fundus von Stoffgeschichten hinsichtlich ihrer rhetorischen Strategien und ihrer Erzählstrukturen analysiert und mit anderen Natur- und Umweltgeschichten verglichen werden (der Wald ist bereits Gegenstand erzähltheoretischer Untersuchungen ${ }^{[38]}$ ). Zum anderen ist das Erzählen von Stoffgeschichten wie jedes niveauvolle Erzählen eine Kunst, die sich nur teilweise in Handlungsanweisungen ausmünzen läßt.

\section{Die Moral der Stoffgeschichte}

Stoffgeschichten haben ein Ziel: Aufklärung über Stoffe und deren Wirkungsformen. Aus den historischen Studien lassen sich Tendenzen ablesen, die Aussagen über die zukünftige Entwicklung einer Stoffgeschichte ermöglichen. Sie gewinnen ihr Gewicht aus der Langzeitbetrachtung und können so etwas wie ein "Gespür" für einen bestimmten Stoff vermitteln. So kann es zu einem Wiedereintritt der Geschichte in sich selbst kommen: Dann nämlich, wenn die Stoffgeschichte zu einem kreativeren und umsichtigeren Umgang mit dem Stoff selbst anregt.

Für Hinweise und Diskussionen danken wir den Teilnehmern an dem WZU-Workshop "Stoffgeschichten" im November 2002: Markus Huppenbauer, Renate Diessenbacher, Riyaz Haider, Simon Meissner und Isabelle Sécher.

\section{Literaturverzeichnis}

[1] Vergleiche S. Böschen: "Katastrophe und institutionelle Lernfähigkeit - Seveso als ambivalenter Wendepunkt der Chemiepolitik", in L. Claussen, E. Geenen, E. Macamo (Ed.) Entsetzliche soziale Prozesse-Theorie und Empirie der Katastrophen, Lit, Münster (2003), p. 139-162 (zugleich: Konflikte, Krisen und Katastrophen - in sozialer und kultureller Sicht, Band 1).

[2] M. Held: Chemiepolitik - Gespräch über eine Kontroverse, $\mathrm{VCH}$, Weinheim (1988)

[3] Vergleiche W. van den Daele: "Der Traum von der 'alternativen' Wissenschaft", Zeitschrift für Soziologie 16 (1987) 403-418.

[4] Vergleiche M. Burke: "Assessing the Environmental Health of Europe", Environmental Science \& Technology 34 (2000) 76A-80A.

[5] H.-J. Schellnhuber: " 'Earth system' analysis and the second Copernican revolution", Nature 402 (1999), Supplement C19-C23; vergleiche aber auch H.-J. Schellnhuber: "Die Koevolution von Natur, Gesellschaft und Wissenschaft - Eine Dreiecksbeziehung wird kritisch", GAIA 10/4 (2001) 258-262.

[6] M. Huppenbauer, A. Reller: "Stoff, Zeit und Energie - Ein transdisziplinärer Beitrag zu ökologischen Fragen", GAIA 5/2 (1996) 103-115.

[7] Vergleiche H. Nowotny, P. Scott, M. Gibbons: Re-Thinking ScienceKnowledge Production in an Age of Uncertainty, Polity Press, Cambridge (2001)

[8] Vergleiche W. Lepenies: Das Ende der Naturgeschichte - Wandel kultureller Selbstverständlichkeiten in den Wissenschaften des 18. und 19. Jahrhunderts, Suhrkamp, Frankfurt am Main (1987); L. Daston: Wunder, Beweise und Tatsachen Zur Geschichte der Rationalität, S. Fischer, Frankfurt am Main (2001)

[9] Vergleiche W. Viehöver: "Diskurse als Narrationen", in R. Keller, A. Hirseland, W. Schneider, W. Viehöver (Ed.): Handbuch Sozialwissenschaftlicher Diskursanalyse, Band 1: Theorien und Methoden, Leske + Budrich, Opladen (2001), p. 177-206.

[10] M. Deneke: " Zur Tragfähigkeit des Stoffwechselbegriffs", in G. Böhme, E. Schramm (Ed.): Soziale Naturwissenschaft - Wege zu einer Erweiterung der Ökologie, Fischer Taschenbuch, Frankfurt am Main (1985), p. 42-52.

[11] K.H. Hörning: Experten des Alltags Die Wiederentdeckung des praktischen Wissens, Velbrück Wissenschaft, Weilerswist (2001), p. 162.

[12] K. Knorr-Cetina: "Laboratorien: Instrumente der Weltkonstruktion", in P. Hoyningen-Huene, G. Hirsch (Ed.): Wozu Wissenschaftsphilosophie?, de Gruyter, Berlin (1988), p. 315-344.

[13] B. Latour: Das Parlament der DingeFür eine politische Ökologie, Suhrkamp, Frankfurt am Main (2001)

[14] M. Polanyi: Implizites Wissen, Suhrkamp, Frankfurt am Main (1966/1985)

[15] H. Collins: Changing Order: Replication and Induction in Scientific Practice, University of Chicago Press, Chicago IL (1992)

[16] H. Collins: "Tacit Knowledge, Trust and the Q of Sapphire", Social Studies of Science 31 (2001) 71-85, insbesondere p. 72.

[17] L. Fleck: Entstehung und Entwicklung einer wissenschaftlichen Tatsache - Einführung in die Lehre vom Denkstil und Denkkollektiv, Suhrkamp, Frankfurt am Main (1935/1983).

[18] S. Böschen: "Wissenschaftsfolgenabschätzung - Über die Veränderung von Wissenschaft im Zuge reflexiver Modernisierung", in S. Böschen, I. Schulz-Schaeffer (Ed.): Wissenschaft in der Wissensgesellschaft, Westdeutscher Verlag, Opladen (2003).

[19] M. Foucault: Die Ordnung des Diskurses, Fischer Taschenbuch, Frankfurt am Main (1970).

[20] S. Funtowicz, J. Ravetz: "The Emergence of
Post-Normal Science", in R. von Schomberg (Ed.): Science, Politics and Morality -

Scientific Uncertainty and Decision Making, Kluwer, Dordrecht (1993), p. 85-123, besonders p. 114f.

[21] W. Bonss, R. Hohlfeld, R. Kollek (Ed.): Wissenschaft als Kontext - Kontexte der Wissenschaft, Junius, Hamburg (1993); insbesondere a) p. 185.

[22] Vergleiche M. Scheringer: Persistence and Spatial Range of Environmental Chemicals, Wiley-VCH, Weinheim (2002).

[23] S. Rowland: "Ozone Hole", in H. Newbold (Ed.): Lifestories - World-renowned Scientists Reflect on their Lives and the Future of Life on Earth, University of California Press, Berkeley CA (2000), p. 134-140, besonders p. $135 \mathrm{f}$

S. Böschen: Risikogenese - Prozesse gesellschaftlicher Gefahrenwahrnehmung. FCKW, Dioxin, DDT und Ökologische Chemie, Leske + Budrich, Opladen (2000).

[24] S. Böschen, M. Scheringer, J. Jaeger: "Wozu Umweltforschung? Über das Spannungsverhältnis zwischen Forschungstraditionen und umweltpolitischen Leitbildern - Teil II: Zum Leitbild 'Reflexive Umweltforschung' ", GAIA 10/3 (2001) 203-212.

[25] A. Reller, M. Braungart, J. Soth, O. von Uexküll: "Silicone - eine vollsynthetische Materialklasse macht Geschichte(n)", GAIA 9/1 (2000) 13-24; insbesondere a) p.16.

[26] S. Tretjakow: Die Arbeit des Schriftstellers, herausgegeben von H. Boehncke, Rowohlt, Reinbek bei Hamburg (1972), p. 81-86.

[27] H.E. Jacob: Sage und Siegeszug des Kaffees - Die Biographie eines weltwirtschaftlichen Stoffes, Rowohlt, Berlin (1934).

[28] J. Dahl: Aufschlüsse: Kalkstein, Feuerstein, Schiefer - drei Versuche zur Geologie, Langewiesche-Brandt, Ebenhausen bei München (1977)

[29] P. Ball: $\mathrm{H}_{2} \mathrm{O}$ - Biographie des Wassers Piper, München (2002).

[30] A. Reller, S. Schmid: Kalk-Kulturgeschichte eines Stoffes, unveröffentlichtes Manuskript, Universität Augsburg (2002).

[31] M. Eichhorn: Kulturgeschichte der "Kulturgeschichten": Typologie einer Literaturgattung, Königshausen \& Neumann, Würzburg (2002).

[32] J.G. Pankau: "Narratio", in G. Ueding (Ed.): Historisches Wörterbuch der Rhetorik, Band 1 , Niemeyer, Tübingen (1992), Sp. 1490-1495.

[33] A. Reller: "Skizze einer Geographie der Ressourcen", Politische Ökologie Nr. 86 (2003) 22-25.

[34] E. Dovifat: " Aktualität - Gegenwartswirkung", in E. Dovifat (Ed.): Handbuch der Publizistik, Band 1: Allgemeine Publizistik, de Gruyter, Berlin (21971), p. 20-28

[35] B. Wessel: "Attentum parare, facere", in G. Ueding (Ed.): Historisches Wörterbuch der Rhetorik, Band 1, Niemeyer, Tübingen (1992), Sp. 1162-1163.

[36] H. Thomae: Das Individuum und seine Welt, Hogrefe, Göttingen (1996).

[37] J. Soentgen: "Feuer, Erde, Wasser, Luft: Die Elemente in der globalen Welt", Sendebeitrag zum Funkkolleg "Glück und Globalisierung" des Hessischen Rundfunks (eine Textversion erscheint 2004 bei Suhrkamp, Frankfurt am Main).

[38] A. Lehmann (Ed.): Der Wald - ein deutscher Mythos?, Reimer, Berlin (2000).

(Eingegangen am 26. November 2003; OS) 